\title{
Análise comparativa de metodologias utilizadas no despacho de caminhões em minas a céu aberto
}

\section{Comparative analysis of the methodologies used to dispatch trucks in open pit mines}

Lásara Fabrícia Rodrigues

Professora, MSc., DEPRO/EM/UFOP

lasara@em.ufop.br

Luiz Ricardo Pinto

Professor, Dr., DEP/EE/UFMG

luiz@dep.ufmg.br

\section{Resumo}

No presente trabalho, realizou-se uma análise comparativa das metodologias utilizadas para o despacho de caminhões em minas a céu aberto. Comparações entre essas metodologias contam com poucos trabalhos publicados na literatura. Metodologias baseadas em programação linear e programação dinâmica e em heurística foram implementadas. Essas metodologias constituem as bases dos algoritmos de alguns dos sistemas de despacho comercializados no Brasil. Foi desenvolvido um modelo de simulação, a fim de se reproduzir o comportamento das operações de lavra de forma a avaliar o impacto das decisões de despacho em cada uma das metodologias estudadas. Os resultados obtidos mostraram o desempenho dos algoritmos, utilizando-se essas metodologias sob diferentes condições em minas a céu aberto. Porém não se pode, através dos resultados obtidos, concluir pela superioridade de uma delas.

Palavras-chave: Despacho de caminhões, minas a céu aberto, simulação.

\begin{abstract}
In the present study a comparative analysis of the methodologies used to dispatch trucks in open pit mines has been carried out. There is a lack of studies comparing these methodologies in literature. Methodology based on linear and dynamic programming, as well as a heuristics-based methodology, have been implemented. These methodologies serve as the basis of algorithms used in some of the dispatch systems sold in Brazil. A simulation model was developed to reproduce the behavior of mining operations in order to evaluate the impact of dispatch decisions in each of the methodologies studied. Results show the performance of algorithms that have used these methodologies under different conditions in open pit mining. However, from results obtained in the present study, it is not possible, to state the superiority of any of them.
\end{abstract}

Keywords: Truck dispatch, open pit mines, simulation. 


\section{Introdução}

As mineradoras realizam suas atividades em minas subterrâneas ou a céu aberto. Em minas a céu aberto, as atividades de carregamento e transporte ocorrem da seguinte maneira: os caminhões se deslocam até a frente de lavra, que são os pontos da mina onde o minério e o estéril estão sendo retirados, são carregados pelos equipamentos de carga e, em seguida, se deslocam para os pontos de descarga. $\mathrm{O}$ minério que sai da mina, também chamado de run of mine (ROM), normalmente é descarregado nos britadores primários, que alimentam as usinas de beneficiamento ou pilhas de homogeneização e o estéril é descarregado nas pilhas de deposição. Muitas vezes é necessário fazer uma blendagem dos minérios de várias frentes de lavra ou de diferentes minas, com o objetivo de se assegurar a uniformidade da alimentação, ação importante sob o ponto de vista operacional (Chanda \& Dagdelen, 1995). Além disso, o teor de cada variável de controle do ROM deve seguir limites de qualidade preestabelecidos, para cada uma delas. Outro parâmetro importante utilizado, para se garantir a correta execução do planejamento de lavra, de forma a garantir que a mina atinja sua vida útil planejada, é a relação estéril/minério (REM), que define a relação entre a quantidade de estéril e de minério que deve ser retirada da mina. Araújo e Souza (2011) afirmam que a vida útil da mina também pode variar em função do avanço da tecnologia. Materiais antes considerados estéril podem, no futuro, ser economicamente aproveitado como minério. Também equipamentos de maior produtividade e softwares mais eficazes podem ser desenvolvidos, exaurindo mais rapidamente a mina.

As minas contam com frotas de equipamentos de carga e transporte para viabilizar a lavra em diferentes frentes. A alocação desses equipamentos depende da sua disponibilidade, da produtividade e da compatibilidade entre os equipamentos de carga e de transporte.

A atividade de transporte de material é um dos mais importantes aspectos na operação de minas a céu aberto (Alarie \& Gamache, 2002). Segundo Tu e Hucka (1985), o custo de transporte representa o maior custo em minas a céu aberto, correspondendo a $50 \%$ dos custos de operação. Alarie e Gamache (2002) afirmam que muitos autores concordam com $\mathrm{Tu}$ e Hucka (1985), mas que alguns deles acreditam que tais custos podem chegar a $60 \%$ dos custos de operação. Logo, uma pequena redução percentual nos custos de transporte resulta em economias significativas (Alarie \& Gamache, 2002). Munirathinam e Yingling (1994) afirmam que, devido a esses altos custos, esforços têm sido feitos para reduzir o custo de transporte. Uma das formas de se otimizar o transporte é a utilização de sistemas de despacho de caminhões e de alocação de equipamentos de carga.

Um sistema de despacho reúne um algoritmo de alocação dinâmica, um algoritmo de sequenciamento de viagens, um sistema de comunicação entre os equipamentos de carga, caminhões e central de comandos (Costa et al., 2004).

Minas a céu aberto utilizam dois critérios para alocar caminhões: alocação estática e dinâmica. A alocação estática fixa os caminhões a um par de pontos de carga e descarga durante um determinado período de tempo. $\mathrm{Na}$ alocação dinâmica, a cada carga e/ou descarga, o caminhão é direcionado pelo sistema de despacho para um ponto específico, de acordo com critérios previamente estabelecidos. Assim, o caminhão pode ser alocado para diferentes pontos, aumentando a produtividade da frota e permitindo que uma frota menor realize as operações (Souza et al., 2010).

A alocação estática ainda é um método utilizado em minas de pequeno e médio porte, isto por não apresentar a obrigatoriedade de utilização de um sistema automático de alocação. Porém esse método proporciona menor produtividade, devido às filas de caminhões e à ociosidade dos equipamentos de carga. Além disso, esse método tende a provocar uma maior variância dos teores do ROM ao longo do tempo, devido ao fato de não haver um controle efetivo e sistemático das descargas dos caminhões.

O objetivo dos sistemas de despacho é a redução dos custos de capital e da operação. Isto se dá através da redução da frota de caminhões necessária, do aumento da produtividade com a utilização dos recursos existentes, do atendimento aos padrões de qualidade da usina de beneficiamento e do aumento da produção da frota. Essas metas são alcançadas através da correta alocação de equipamentos de carga e de transporte em tempo real, monitoramento e determinação de decisões de alocação. Tais procedimentos aumentam a utilização de recursos e diminuem o tempo de espera em todos os caminhos.

Quando se utiliza a alocação dinâmica de caminhões e, consequentemente, um sistema de despacho, existem diferentes metodologias que podem ser usadas como estratégia de decisão de alocação. Esse trabalho objetiva comparar algumas dessas metodologias, sob diferentes condições (cenários). Segundo Munirathinam e Yingling (1994), comparações entre essas metodologias contam com poucos estudos publicados na literatura. Para se realizar essa comparação, foi desenvolvido um modelo de simulação, a fim de se reproduzir o comportamento das operações de lavra, de forma a se avaliar o impacto das decisões de despacho, em cada uma das metodologias estudadas.

A simulação computacional foi utilizada por não ser possível encontrar, em uma mesma mina, diferentes metodologias aplicadas. Segundo Ingalls (2008), a simulação é um processo de se conceber um modelo de um sistema real e de se conduzirem experimentos com esse modelo, possibilitando-se avaliar diferentes estratégias para a operação do mesmo. Tu e Hucka (1985) e Maran e Topuz (1988) afirmam que a simulação pode ser usada para experimentação e avaliação de alocação de caminhões e problemas de despacho, especialmente quando métodos analíticos não são apropriados. Além disso, a simulação pode ser usada para reproduzir a aleatoriedade de problemas em equipamentos e variações no tempo de viagens e processos, tornandose uma ferramenta adequada para se checar a viabilidade de um plano (Fioroni et al., 2008).

\section{Descrição das metodologias avaliadas para alocação dinâmica}

As metodologias apresentadas, nessa seção, são as bases de alguns sistemas de despacho que funcionam no Brasil. É evidente que os sistemas comerciais podem apresentar variações dessas metodologias, devido à própria evolução dos mes- mos. Além disso, os algoritmos, que são usados em tais sistemas, normalmente não são públicos devido a razões comerciais. 


\section{Metodologia baseada em programação linear e programação dinâmica}

A metodologia baseada em programação linear e programação dinâmica utilizou, como referência, os trabalhos desenvolvidos por White et al. (1982), White et al. (1993), White e Olson (1986) e Pinto e Merschmann (2001). O modelo aqui utilizado contempla, ainda, res-

\section{Modelo de programação linear}

O modelo de programação linear determina o fluxo ótimo de material entre os pontos de carga e os pontos de descarga, minimizando o custo de remanuseio e estocagem, obedecendo a limites de qualidade, para cada ponto de descar- trições desenvolvidas para torná-lo mais realista, como a relação estéril/minério, a produção mínima em cada usina de beneficiamento e a possibilidade de utilização de mais de um ponto de descarga (usina de beneficiamento).

Essa metodologia utiliza um mode- lo de programação linear, para se determinar o fluxo ótimo entre cada ponto de carga e de descarga (caminhos) e um modelo de programação dinâmica, para se alocarem os caminhões para o caminho selecionado previamente pela programação linear. ga, além de satisfazer as necessidades de alimentação da usina.

A solução do modelo depende da configuração de seus parâmetros, que pode variar de tempos em tempos. Para se configurar o modelo, de- vem ser observadas as condições então reinantes, como as frentes de lavra disponíveis, os limites de qualidade desejáveis para o ROM, a relação estéril/minério desejável, etc. $\mathrm{O}$ modelo é descrito a seguir.

$$
\begin{gathered}
\operatorname{Min} \sum_{i=1}^{Q} \sum_{k=1}^{D}\left(C_{m} Q_{i k}\right)+C_{P}\left(\sum_{k=1}^{D} P T_{k}-\sum_{i=1}^{Q+S} \sum_{k=1}^{D} Q_{i k}\right)+\sum_{i=1}^{S} \sum_{k=1}^{D}\left(C_{s} Q_{i k}\right) \sum_{i=1}^{Q+S} \sum_{j=1}^{V} \sum_{k=1}^{D}\left(L_{i j} C_{q} X_{i j} Q_{i k}\right) \\
\text { s.a.: } L_{j k} \leq L A_{j k}+\sum_{i=1}^{Q+S}\left(X_{i j}-L A_{j k}\right) Q_{i k} T C_{k} /\left(M C_{k} / S G\right) \quad \forall j \in V, \forall K \in U \\
L S_{j k} \geq L A_{j k}+\sum_{i=1}^{Q+S}\left(X_{i j}-L A_{j k}\right) Q_{i k} T C_{k} /\left(M C_{k} / S G\right) \quad \forall j \in V, \forall K \in U \\
R_{i} \geq \sum_{k=1}^{D} Q_{i k} \quad \forall i \in Q \cup S \\
P T_{k} \geq \sum_{i=1}^{Q+S} Q_{i k} \quad \forall k \in U \\
\sum_{i=1}^{M} Q_{i k} \geq P M i n_{k} \quad \forall k \in U \\
\sum_{i=1}^{E} \sum_{k=1}^{P} Q_{i k} \geq R E M \sum_{i=1}^{M} \sum_{k=1}^{U} Q_{i k} \\
\sum_{i=1}^{E} \sum_{k=1}^{P} Q_{i k} \geq 1.1 * R E M \sum_{i=1}^{M} \sum_{k=1}^{U} Q_{i k} \\
Q_{i k} \geq 0 \quad \forall i \in Q \cup S \forall k \in D
\end{gathered}
$$

\section{Conjuntos:}

M: Conjunto das frentes de lavra de minério.

E: Conjunto das frentes de lavra de estéril.

Q: Conjunto de equipamentos de carga alocados nas frentes de lavra (M U E).

U: Conjunto das usinas de beneficiamento.

P: Conjunto das pilhas de estéril.

D: Conjunto dos pontos de descarga (U U P).

V: Conjunto das variáveis de qualidade analisadas.

S: Conjunto de equipamentos de carga alocados nas pilhas de estoque. Parâmetros:

$\mathrm{C}_{\mathrm{m}}$ : Custo de movimentação de material $\left(\mathrm{h} / \mathrm{m}^{3}\right)$.

$\mathrm{C}_{\mathrm{s}}$ : Custo de estocagem de material $\left(\mathrm{h} / \mathrm{m}^{3}\right)$.

$\mathrm{C}_{\mathrm{q}}$ : Custo associado à qualidade do minério $\left(\mathrm{h} / \mathrm{m}^{3}\right)$.

$\mathrm{C}_{\mathrm{p}}^{\mathrm{q}}$ : Custo associado à alimentação da usina de beneficiamento $\left(\mathrm{h} / \mathrm{m}^{3}\right)$.

$\mathrm{R}_{:}^{\mathrm{p}}$ : Taxa-limite de carregamento, no ponto $i\left(\mathrm{~m}^{3} / \mathrm{h}\right)$.

$\mathrm{L}_{\mathrm{j} \mathrm{k}}$ : Importância da variável $j$, no ponto de descarga $k\left(\mathrm{~L}_{\mathrm{jk}}=1\right.$, se baixo e $\mathrm{L}_{\mathrm{jk}}=-1$, se alto).

$\mathrm{X}_{\mathrm{i}:}$ : Teor da variável $j$ no minério do ponto de carga $i(\%)$.

$\mathrm{PT}_{\mathrm{k}}$ : Taxa de alimentação máxima admissível nas usinas (ponto de descarga) $k\left(\mathrm{~m}^{3} / \mathrm{h}\right)$.

$\mathrm{PMin}_{\mathrm{k}}$ : Taxa de alimentação mínima admissível na usina (ponto de descarga) $k\left(\mathrm{~m}^{3} / \mathrm{h}\right)$.

$\mathrm{MC}_{\mathrm{k}}$ : Massa de controle, no ponto de descarga $k(\mathrm{t})$.

SG: Peso específico do material $k\left(t / \mathrm{m}^{3}\right)$.

$\mathrm{TC}_{\mathrm{k}}$ : Intervalo de controle, no ponto de descarga $k(\mathrm{~h})$.

$\mathrm{LI}_{\mathrm{j} \mathrm{k}}$ : Limite inferior para o teor da variável $j$, no ponto de descarga $k(\%)$.

$\mathrm{LA}_{\mathrm{j} \mathrm{k}}$ : Teor corrente para a variável $j$ no ponto de descarga $k(\%)$. $\mathrm{LS}_{\mathrm{jk}}$ : Limite superior, para o teor da variável $j$, no ponto de descarga $k(\%)$.

Variáveis de decisão:

$\mathrm{Q}_{\mathrm{i}}$ : Quantidade de material que sai do ponto de carga $i$ e vai para o ponto de descarga $k\left(\mathrm{~m}^{3} / \mathrm{h}\right)$. 
A função objetivo é apresentada na Equação (1) e representa o custo total, composto pelos custos de movimentação, de capacidade não utilizada da usina, de estocagem e de qualidade. Segundo White e Olson (1986), os custos não representam os custos reais, ou seja, são arbitrários e devem obedecer à seguinte ordem crescente: custo de movimentação $\left(C_{m}\right)$, custo de qualidade $\left(C_{q}\right)$, custo de estocagem $\left(C_{s}\right)$ e custo de alimentação da usina $\left(C_{p}\right)$. As restrições são apresentadas pelas Equações de (2) a (9). As restrições (2) e (3) garantem que as variáveis controladas estejam dentro dos limites de qualidade da mistura. A

\section{Modelo de programação dinâmica}

Na programação dinâmica, a cada necessidade de alocação, verifica-se, para cada um dos possíveis caminhos, qual será a melhor opção de alocação, ou seja, o caminho que requer uma alocação de caminhão mais rapidamente. $\mathrm{O}$ caminhão alocado deverá chegar, ao ponto de

\section{Onde:}

$\mathrm{X}_{\mathrm{i}}$ : Horário que cada caminho $i$ requer uma alocação de caminhão (h).

$\mathrm{H}_{\mathrm{io}}$ : Capacidade do caminhão alocado para o transporte $\left(\mathrm{m}^{3}\right)$.

$\mathrm{P}_{\mathrm{i}}$ : Fluxo de material, no caminho $i\left(\mathrm{~m}^{3} / \mathrm{h}\right)$. $\mathrm{L}$ : Horário que o último caminhão foi alocado, para o caminho $i(\mathrm{~h})$.

$\mathrm{T}_{\mathrm{i}}$ : Tempo de viagem, para o caminho $i(\mathrm{~h})$.

O modelo de programação dinâmi$c a$ aloca caminhões a caminhos considerando a cada alocação apenas o tempo

\section{Metodologia baseada em heurística}

A metodologia baseada em heurística utilizou, como referência, o trabalho de Pinto (2004). Essa heurística fundamenta-se na combinação de um conjunto de estratégias ou regras de despacho utilizadas para realizar a alocação de forma a melhorar o resultado. Essas estratégias são Qualidade, Ritmo de Lavra, Ritmo de Produção, Tempo de Fila e Tempo de Ciclo. Entre elas, as três primeiras são estratégias voltadas para a redução da variância dos teores do ROM e as duas últimas são estratégias que objetivam o aumento da produtividade das frotas de carregamento e transporte via redução de tempos ociosos.

A escolha entre essas estratégias é restrição (4) assegura que a quantidade retirada do ponto de carga seja menor ou igual à produção do equipamento de carga alocado para aquele ponto de carga. A restrição (5) determina que a quantidade total retirada dos pontos de carga não exceda a capacidade máxima de cada usina. A restrição (6) garante que a produção será maior ou igual a uma produção mínima necessária para a alimentação da usina. As restrições (7) e (8) garantem que a REM seja cumprida dentro de um limite aceitável, que aqui ficou estabelecido em $10 \%$. A restrição (9) assegura que o ritmo de carregamento dos pontos de carga não

carga, quando o carregamento dos caminhões alocados anteriormente estiver terminando. Caso isso não seja possível, o caminhão será alocado de modo que permaneça o menor tempo possível em fila. Logo, esse procedimento aloca caminhões a caminhos de modo a reduzir

$$
\mathrm{X}_{\mathrm{i}}=\mathrm{L}_{\mathrm{i}}+\left(\mathrm{H}_{\mathrm{io}} / \mathrm{P}_{\mathrm{i}}\right)-\mathrm{T}_{\mathrm{i}}
$$

em fila gerado pela alocação que está sendo feita (alocação corrente), ou seja, não realiza a alocação tendo, como base, uma visão global do problema. Por esse motivo, Alarie e Gamache (2002) e Alvarenga (1997) consideram que, como esse modelo de programação dinâmica não despacha de acordo com uma visão global do problema de despacho, ele pode ser considerado um método heurístico.

Além disso, esse procedimento incorpora simplificações, em relação às

baseada na experiência dos operadores do sistema, nas características da mina e nas necessidades de curto prazo. $\mathrm{Mu}-$ nirathinam e Yingling (1994) afirmam que nenhuma das estratégias de alocação predomina sobre as demais e que todas dependem do estado da mina. As estratégias Tempo de Fila e Tempo de Ciclo, normalmente, devem ser utilizadas quando a mina apresenta frentes cujos teores não são excessivamente heterogêneos ou que possuam pilhas de homogeneização. As estratégias Qualidade, Ritmo de Lavra e Ritmo de Produção se aplicam a situações onde a qualidade do ROM é imprescindível e quando ocorre grande variabilidade, no teor das variáveis entre assuma valores negativos.

$\mathrm{O}$ modelo deve ser solucionado sempre que ocorram mudanças na configuração da mina, ou seja, nos parâmetros do modelo, porque mudanças na configuração levam a mudanças na solução-ótima. Isso se justifica, uma vez que o modelo leva em consideração a configuração corrente da mina.

Através desse modelo, é obtido o fluxo ótimo de cada ponto de carga $i$, para cada ponto de descarga $k\left(Q_{i k}\right)$. Esse fluxo é utilizado como dado de entrada no modelo de programação dinâmica, porém, nesse último, é denominado fluxo no caminho $i\left(P_{i}\right)$.

ao máximo o tempo em fila.

Esse modelo utiliza a Equação (10) para calcular o horário que cada caminho $i$ requer uma alocação de um caminhão $\left(X_{i}\right)$. O caminhão é alocado ao caminho que necessitar primeiro dessa alocação, ou seja, para o menor valor de $X_{i}$.

situações encontradas na prática. Entre elas, pode-se citar a utilização de valores médios, para os tempos de deslocamento, carga e descarga, ao invés das distribuições de probabilidade que regem tais fenômenos. A utilização de valores médios, quando se têm atividades em série, como aqui (deslocamento vazio seguido de carga, seguido de deslocamento carregado, seguido de descarga), pode subestimar o tempo total de ciclo, ou seja, a soma das médias não é igual à média total.

as diversas frentes.

A metodologia baseada em heurística funciona da seguinte maneira: a primeira estratégia utilizada, em todas as possíveis combinações, é a estratégia Ritmo de Produção. Em seguida, ordenam-se as demais estratégias de alocação. A ordenação dessas estratégias é utilizada quando duas ou mais frentes ficam empatadas na primeira estratégia escolhida. Nesse caso, passa-se para a segunda estratégia e, se o empate persistir, passa-se para a terceira e assim por diante. Geralmente, o desempate ocorre ainda na segunda estratégia. $\mathrm{O}$ pseudocódigo e maiores detalhes sobre esse procedimento heurístico e sobre as 
estratégias Ritmo de Produção, Qualidade, Ritmo de Lavra, Tempo de Fila e Tempo de Ciclo podem ser encontrados em Rodrigues (2006).

A estratégia Ritmo de Produção é utilizada quando existe mais de um ponto de descarga de minério. Nessa estratégia, é calculado o desvio do ritmo de produção real em relação ao ritmo de produção ideal, para cada ponto de descarga. Em seguida, escolhe-se o ponto de descarga que apresentar o maior desvio. Caso sejam obtidos valores iguais, para o desvio, em mais de um ponto de descarga, escolhe-se, aleatoriamente, um dos pontos de descarga.

A estratégia Qualidade objetiva manter as variáveis dentro dos limites de qualidade em tempo real. Para isso, divide-se a produção em lotes, na tenta-

\section{Implementação computacional}

A implementação da metodologia baseada em programação linear e programação dinâmica utilizou o software de otimização Lingo 7.0, para resolver o problema de programação linear, e o software Borland Delphi 5.0, para resolver a programação dinâmica. A metodologia baseada em heurística utilizou o software Borland Delphi 5.0, para programar cada uma das estratégias de despacho. A simulação foi realizada utilizando-se o software Borland Delphi 5.0 e as rotinas do SIMIN - Simulador para Mineração (Pinto, 1999), para simular os modelos. O SIMIN foi utilizado para comparar os modelos em diferentes cenários de produção, já que não é possível encontrar, na realidade, diferentes metodologias aplicadas numa mesma mina.

\section{Resultados obtidos}

As simulações foram rodadas utilizando-se 10 corridas, cada uma com duração de 4320 minutos. Para a comparação da metodologia baseada em $\mathrm{Heu}$ rística, foram ordenadas as estratégias de despacho, pois, como já foi mencionado, caso ocorra empate em uma das estratégias, a estratégia seguinte é utilizada como desempate. Em cada um dos cenários analisados, foi estabelecido que:

Situação A - Heurística com ordem das estratégias de despacho Ritmo de Produção, Ritmo de Lavra, Qualidade dos Lotes e Tempo de Fila.

Situação B - Heurística com ordem das estratégias de despacho Ritmo de tiva de colocar a qualidade de cada lote dentro desses limites. Então, para cada alternativa de frente, para a qual o caminhão pode ir, após sua descarga, é calculado, para cada variável, o desvio que aquela alocação provocaria, no lote corrente. $\mathrm{O}$ caminhão será despachado para a frente que apresentar o menor somatório dos desvios.

A estratégia Ritmo de Lavra visa manter os ritmos de lavra, de cada frente, o mais próximo possível dos valores ideais. Quando se utiliza essa estratégia, calcula-se, para cada frente, o desvio de sua produção real, em relação à sua produção ideal. A frente escolhida é aquela que apresentar o maior desvio, ou seja, a que estiver mais defasada em relação à produção ideal.

Na estratégia Tempo de Fila, após

Uma mina virtual foi desenvolvida com o objetivo de reproduzir os diferentes cenários encontrados em minas a céu aberto. $\mathrm{O}$ objetivo dessas análises foi comparar as metodologias sob condições diferentes, ou seja, comparar as metodologias nas diversas situações encontradas nas minas a céu aberto. As principais características de cada cenário são descritas abaixo e maiores detalhes podem ser encontrados em Rodrigues (2006).

O Cenário 1 é composto por 4 frentes, sendo uma de estéril e 3 de minério, 2 usinas de beneficiamento e uma única pilha de estéril. As frentes de minério não atendem às necessidades da usina de beneficiamento isoladamente, ou seja, é necessário misturar minério das frentes

Produção, Ritmo de Lavra, Tempo de Fila e Qualidade dos Lotes.

Situação C - Heurística com ordem das estratégias de despacho Ritmo de Produção, Tempo de Fila, Qualidade dos Lotes e Ritmo de Lavra.

Situação D - Heurística com ordem das estratégias de despacho Ritmo de Produção, Tempo de Fila, Ritmo de Lavra e Qualidade dos Lotes.

Situação E - Heurística com ordem das estratégias de despacho Ritmo de Produção, Qualidade dos Lotes, Tempo de Fila e Ritmo de Lavra.

Situação F - Heurística com ordem das estratégias de despacho Ritmo de a descarga do caminhão, o sistema calcula os prováveis tempos de fila, caso o caminhão seja enviado para cada uma das frentes e pontos de descarga. O tempo de fila total é a soma do tempo de fila na frente com o tempo de fila no ponto de descarga. Ordena-se o tempo de fila total em ordem crescente e despacha-se o caminhão para o par de pontos de carga e de descarga que proporcionar o menor tempo de fila total.

Na estratégia Tempo de Ciclo, após a descarga do caminhão, calcula-se o instante de retorno do caminhão ao ponto de descarga. O tempo de ciclo é a diferença entre o instante estimado de retorno do caminhão ao ponto de descarga e o instante corrente. Em seguida, escolhe-se o trajeto que apresentar o menor instante de retorno do caminhão ao ponto de descarga.

para atender as restrições de qualidade impostas pela usina.

O Cenário 2 diferencia-se do Cenário $1 \mathrm{em}$ relação a quantidade de caminhões, ou seja, no Cenário 1 são utilizados 15 caminhões e, no Cenário 2, são utilizados 22 caminhões.

No Cenário 3, cada uma das frentes atende às necessidades da usina de beneficiamento isoladamente, ou seja, retirar minério de uma ou mais frentes é indiferente em relação aos limites de qualidade estabelecidos pela usina.

O Cenário 4 apresenta as mesmas características do Cenário 3, exceto quanto ao número de caminhões utilizados para o transporte. O Cenário 3 utiliza 15 caminhões e o Cenário 4 utiliza 22 caminhões.

Produção, Qualidade dos Lotes, Ritmo de Lavra e Tempo de Fila.

Situação G - Programação linear e programação dinâmica.

As situações A, B, C, D, E e F são referentes à metodologia baseada em heurística e a Situação $\mathrm{G}$, à metodologia baseada em programação linear e programação dinâmica.

A heurística com a estratégia de despacho Tempo de Ciclo não foi utilizada, devido a sua baixa adoção, em situações reais. Essa estratégia resulta na superutilização dos equipamentos de carga das frentes mais próximas e na subutilização dos demais. 
Os resultados obtidos nos Cenários 1, 2, 3 e 4 são mostrados na Tabela 1. Esses resultados mostraram que, em todos os cenários, as Situações A e B, Situações C e D e Situações E e F apresentaram os mesmos resultados entre si. Logo, ob- servou-se que não ocorreu empate entre duas ou mais frentes na segunda estratégia escolhida.

\begin{tabular}{|c|c|c|c|c|c|}
\hline & & Produção de Minério ( $t$ ) & Produção Total (t) & REM & Desvio (\%) \\
\hline \multirow{7}{*}{ Cenário 1} & Situação A & 328.820 & 440.010 & 33,81 & 0,038 \\
\hline & Situação B & 328.820 & 440.010 & 33,81 & 0,038 \\
\hline & Situação C & 328.670 & 439.690 & 33,77 & 0,63 \\
\hline & Situação D & 328.670 & 439.690 & 33,77 & 0,63 \\
\hline & Situação E & 330.670 & 442.340 & 33,77 & 0,002 \\
\hline & Situação F & 330.670 & 442.340 & 33,77 & 0,002 \\
\hline & Situação G & 328.560 & 444.710 & 35,35 & 0 \\
\hline \multirow{7}{*}{ Cenário 2} & Situação A & 370.830 & 502.970 & 35,63 & 0,15 \\
\hline & Situação B & 370.830 & 502.970 & 35,63 & 0,15 \\
\hline & Situação C & 463.090 & 619.620 & 33,80 & 0,62 \\
\hline & Situação D & 463.090 & 619.620 & 33,80 & 0,62 \\
\hline & Situação E & 345.600 & 470.670 & 36,19 & 0,0002 \\
\hline & Situação F & 345.600 & 470.670 & 36,19 & 0,0002 \\
\hline & Situação G & 345.690 & 476.170 & 37,74 & 0,0004 \\
\hline \multirow{7}{*}{ Cenário 3} & Situação A & 328.820 & 440.010 & 33,81 & 0 \\
\hline & Situação B & 328.820 & 440.010 & 33,81 & 0 \\
\hline & Situação C & 328.670 & 439.690 & 33,78 & 0 \\
\hline & Situação D & 328.670 & 439.690 & 33,78 & 0 \\
\hline & Situação E & 173.010 & 241.220 & 39,42 & 0 \\
\hline & Situação F & 173.010 & 241.220 & 39,42 & 0 \\
\hline & Situação G & 172.780 & 243.970 & 41,20 & 0 \\
\hline \multirow{7}{*}{ Cenário 4} & Situação A & 370.830 & 502.970 & 35,63 & 0 \\
\hline & Situação B & 370.830 & 502.970 & 35,63 & 0 \\
\hline & Situação C & 463.090 & 619.620 & 33,80 & 0 \\
\hline & Situação D & 463.090 & 619.620 & 33,80 & 0 \\
\hline & Situação E & 172.910 & 250.240 & 44,72 & 0 \\
\hline & Situação F & 172.910 & 250.240 & 44,72 & 0 \\
\hline & Situação G & 172.740 & 253.160 & 46,55 & 0 \\
\hline
\end{tabular}

Os cenários 1 e 3 e 2 e 4, respectivamente, apresentam, nas Situações A e B, $\mathrm{C}$ e $\mathrm{D}$, os mesmos valores para a produção de minério e total. A diferença entre esses cenários é que, nos cenários 1 e 2 as frentes não atendem isoladamente às necessidades da usina de beneficiamento e, nos cenários 3 e 4 atendem. Nas Situações A, B, C e D, a estratégia Qualidade dos Lotes é a terceira ou quarta estratégia e, como já discutido, não chegou a ser utilizada. Logo, nessas Situações, as estratégias utilizadas foram Ritmo de Lavra e Tempo de Fila, estratégias estas que não são afetadas por diferenças de qualidade.

A ocorrência de produção menor, quando se adota a estratégia de despacho Tempo de Fila (Situações C e D), prioritária em relação à estratégia de despacho Ritmo de Lavra (Situações A e B), nos cenários 1 e 3 , pode ser atribuída à existência de uma frente de minério muito distante da usina (a frente 4). O envio de caminhões para essa frente, mesmo implicando um tempo de fila menor, pode causar uma menor produção, pois o tempo de ciclo total, para essa frente, é muito alto. Esse efeito se anula, quando o número de caminhões é aumentado (Cenários 2 e 4).

A relação estéril/minério (REM) foi atendida em todos os cenários analisados, utilizando-se as diferentes metodologias, ou seja, todas obtiveram valores superiores a $33 \%(0,33)$. Entretanto, em alguns cenários (Cenário 2 - Situação G; Cenário 3 - Situações E, F e G; Cenário 4 - Situações E, F e G) a REM apresentou valores altos. A Situação $\mathrm{G}$ estabelece, na Programação Linear, um limite superior para a REM (Equação 8). Entretanto, na Programação Dinâmica, o caminhão é alocado ao ponto de carga que lhe proporcionar o menor tempo em fila, ou seja, o caminhão não é alocado de acordo com uma visão global do problema. As outras Situações (A, B, C, D, E e F) que são baseadas em heurísticas não estabelecem, diretamente, um limite para a REM, ou seja, os resultados obtidos para a REM representam resultado das regras adotadas, em cada uma das estratégias de despacho.
Tabela 1

Resultados obtidos nos cenários.

O desvio, em relação aos limites de qualidade (Desvio) mostrados na Tabela 1 , em percentual (\%), representa o quão distante as variáveis de controle estão dos seus limites preestabelecidos. Nos Cenários 3 e 4, todos os desvios foram nulos, ou seja, a produção obtida permaneceu dentro dos limites de qualidade. Esses resultados eram esperados, já que, nesses cenários, cada uma das frentes atende aos limites de qualidade isoladamente. Nos demais cenários, ocorreram desvios em relação aos limites de qualidade e, nas Situações que utilizam heurística, os menores desvios, em cada cenário, aconteceram nas Situações E e F. Esses resultados podem ser explicados em função da segunda estratégia de despacho utilizada, nessas Situações, ser a Qualidade dos Lotes.

Nos cenários 2 e 4, as Situações C e $\mathrm{D}$ apresentaram os melhores resultados, em relação à produção de minério e à produção total. Esses resultados podem ser explicados pelo aumento no número de caminhões disponíveis, nesses cenários, em relação aos cenários 1 e 3 , respectivamente. Tais resultados também 
podem ser explicados pelo fato de a estratégia Tempo de Fila ser uma estratégia voltada para a produtividade da mina e ser a segunda estratégia de despacho nesses cenários.

No cenário 1, as Situações E e F apresentaram os melhores resultados, em termos de produção de minério, e as Situação $\mathrm{G}$, em relação à produção total. No

\section{Conclusão}

O trabalho apresenta os resultados de uma análise comparativa de metodologias utilizadas para o despacho de caminhões em minas a céu aberto. Comparações entre essas metodologias são pouco contempladas nos trabalhos publicados na literatura. E, além disso, esses algoritmos têm sido cada vez mais utilizados em minas de diferentes portes, isto em função de eles contribuírem para a redução do custo da operação de transporte, aumentarem a produtividade da frota e melhorarem o aproveitamento dos recursos minerais.

A simulação foi utilizada, nessa análise, para reproduzir o comportamento das operações de lavra. As metodologias baseadas em programação linear e programação dinâmica, bem como a baseada em heurísticas foram implementadas e testadas sob diferentes condições. Os cenários analisados utilizaram frentes que atendiam, isoladamente, às necessidades das usinas de be-

\section{Agradecimentos}

Os autores agradecem à CAPES pelo apoio ao desenvolvimento desse

\section{Referências bibliográficas}

cenário 3, as Situações A e B apresentaram os melhores resultados, em termos de produção de minério e produção total.

Nos cenários 3 e 4, as Situações E, $\mathrm{Fe} \mathrm{G}$ apresentam valores muito inferiores às demais situações, em relação à produção de minério e total. $\mathrm{O}$ caso das Situações $\mathrm{E}$ e $\mathrm{F}$ pode ser explicado devido às frentes não atenderem isoladamente, nes-

neficiamento (Cenários 3 e 4) e cenários que não atendiam (Cenários 1 e 2). Esses cenários incluíam, também, variação no número de caminhões disponíveis para o transporte.

As metodologias testadas, nesse estudo, apresentaram resultados adequados de acordo com os testes realizados. Entretanto nenhuma delas produziu resultados significativamente melhores que as outras, nos diferentes cenários analisados, comprovando que nenhuma estratégia de alocação predomina em relação às demais e que todas dependem do estado da mina. Esses resultados confirmam que a estratégia de despacho utilizada depende de outros parâmetros ligados aos sistemas de despacho, tais como custo, sofisticação tecnológica no tocante ao rastreamento dos veículos (alguns sistemas rastreiam os equipamentos via satélite, outros não), quantidade de informações extras disponibilizadas pelos sistemas (como, ses cenários, às necessidades das usinas e devido a estratégia Qualidade dos Lotes estar em segundo lugar, em relação às demais estratégias de despacho. No caso da Situação G, os limites de controle das variáveis são estabelecidos pela Programação Linear, mas a Programação Dinâmica aloca o caminhão ao ponto de carga que resultar no menor tempo em fila.

por exemplo, controle de funções dos equipamentos, controle de parâmetros de manutenção) e tamanho de frota (alguns sistemas têm tempo de resposta muito alto quando o número de equipamentos é grande).

Logo, a comparação entre essas metodologias auxilia no processo de decisão da estratégia de despacho a ser utilizada em situações práticas em minas a céu aberto. O tomador de decisão, com base na configuração da mina, naquele momento, e nas suas necessidades de curto prazo, pode utilizar a estratégia de despacho que mais contribua para alcançar objetivos, como aumentar a produção ou reduzir a variância dos teores do ROM. Além disso, essa comparação pode auxiliar o gestor em decisões sobre aquisição de sistemas de despacho, pois as metodologias comparadas são as bases de alguns dos sistemas de despacho comercializados no Brasil. trabalho e aos revisores anônimos pelos valiosos comentários e sugestões.

ALARIE, S., GAMACHE, M. Overview of Solution Strategies Used in Truck Dispatching Systems for Open Pit Mines. International Journal of Surface Mining, Reclamation and Environment, v. 16, p. 59-76, 2002.

ALVARENGA, G. B. Despacho ótimo de caminhões numa mineração de ferro utilizando algoritmo genético com processamento paralelo. Belo Horizonte: Programa de Pós-Graduação em Engenharia Elétrica, UFMG, 1997. 80 p. (Dissertação de Mestrado).

ARAÚJO, F. C. R., SOUZA, M. J. F. Uma heurística para o planejamento operacional de lavra com alocação dinâmica de caminhões. REM - Revista Escola de Minas, v. 64, n. 1, p. 69-76, 2011.

CHANDA, E. K. C., DAGDELEN, K. Optimal blending of mine production using goal programming and interactive graphics systems. International Journal of Surface Mining, Reclamation and Environment, v. 9, p. 203-208, 1995.

COSTA, F. P., SOUZA, M. J. F., PINTO, L. R. Um modelo de alocação dinâmica de caminhões. Brasil Mineral, v. 231, p. 26-31, 2004.

INGALLS, R. G. Introduction to simulation. In: WINTER SIMULATION CONFERENCE, Proceedings... Miami, USA, 2008. 
FIORONI, M. M., FRANZESE, L. A. G., BIANCHI, T. J., EZAWA, L., PINTO, L. R., MIRANDA Jr, G. Concurrent simulation and optimization models for mining planning. In: WINTER SIMULATION CONFERENCE, Proceedings... Miami, USA, 2008.

MARAN, J., TOPUZ, E. Simulation of truck haulage systems in surface mines. International Journal of Surface Mining, v. 2, p. 43-49, 1988.

MUNIRATHINAM, M., YINGLING, J. C. A review of computer-based truck dispatching strategies of surface mining operations. International Journal of Surface Mining, Reclamation and Environment, v. 8, p. 1-15, 1994.

PINTO, L. R. Metodologia de análise do planejamento de lavra em minas a céu aberto baseada em simulação das operações de lavra. Rio de Janeiro: Programa de Engenharia de Produção - COPPE, UFRJ, 1999. (Tese de Doutorado).

PINTO, L. R. Uso de técnicas de pesquisa operacional na otimização das operações de carregamento e transporte em minas a céu aberto. Belo Horizonte: Departamento de Engenharia de Produção, UFMG, 2004, 107 p. (Notas de Aula de Curso de Extensão).

PINTO, L. R., MERSCHMANN, L. H. C. Planejamento operacional da lavra de mina usando modelos matemáticos. REM - Revista Escola de Minas, v. 54, n.3, p. 211-214, 2001.

RODRIGUES, L. F. Análise comparativa de metodologias utilizadas no despacho de caminhões em minas a céu aberto. Belo Horizonte: Programa de Pós-Graduação em Engenharia de Produção, UFMG, 2006. 103 p. (Dissertação de Mestrado).

SOUZA, M. J. F., COELHO, I. M., RIBAS, S., SANTOS, H. G., MERSCHMANN, L. H. C. A hybrid heuristic algorithm for the open-pit-mining operational planning problem. European Journal of Operational Research, v. 207, p. 1041-1051, 2010.

TU, J. H., HUCKA, V. J. Analysis of open-pit truck haulage system by use of a computer model. CIM Bulletin, v. 78, n. 879, p. 53-59, 1985.

WHITE, J. W., OLSON, J. P., VOLNOUT, S. I. On improving truck/shovel productivity in open pit mines. CIM Bulletin, p. 43-49, 1993.

WHITE, J. W., ARNOLD, M. J., CLEVENGER, J. G. Automated open-pit truck dispatching at Tyrone. Engineering and Mining Journal, v.183, n. 6, p. 76-84, 1982.

WHITE, J. W., OLSON, J. P. Computer-based dispatching in mines with concurrent operating objectives. Mining Engineering, v. 38, n. 11, p. 1045-1054, 1986.

Artigo recebido em 17 de maio de 2011. Aprovado em 09 de março de 2012. 